\title{
Cellular Automata Based Temporal Process Understanding of Urban Growth
}

\author{
Jianquan Cheng, Ian Masser \\ Urban Planning and Management Division, International Institute for Geo-Information Sci- \\ ence and Earth Observation (ITC), Hengelosestraat 99, P.O.Box 6, 7500 AA, Enschede, \\ The Netherlands \\ \{Jianquan, Masser\}@itc.n]
}

\begin{abstract}
Understanding of urban growth process is highly crucial in making development plan and sustainable growth management policy. As the process involves multi-actors, multi-behavior and various policies, it is endowed with unpredictable spatial and temporal complexities, it requires the occurrence of new simulation approach, which is process-oriented and has stronger capacities of interpretation. In this paper, A cellular automata-based model is designed for understanding the temporal process of urban growth by incorporating dynamic weighting concept and project-based approach. We argue that this methodology is able to interpret and visualize the dynamic process more temporally and transparently.
\end{abstract}

\section{Introduction}

The city is a typical complex system, which is characterized with a self-organization property $[1,2]$. Understanding of urban development process is highly crucial in urban development planning and sustainable growth management. Urban development process involves multi-actors, multi-behaviors and various policies, which results in their spatial and temporal complexities. Due to the hidden complexity of reality, our science has become less orientated to prediction but more an aid to understanding, to structure debate [3]. Couclelis [4] first put forward the idea of spatial understanding support system (SUSS). Proper understanding of complex system is the prerequisite to its prediction.

Cellular automata (CA), a technique developed recently, has been receiving more and more attention in GIS modeling due to its simplicity, transparency, strong capacities for dynamic spatial simulation, and innovative bottom-up approach. Numerous literatures can be seen even in the field of urban growth CA modeling on various scales (regional, municipal and town) e.g. [5-9].

In contrast to classic $\mathrm{CA}$, more and more modifications have been made to improve its modeling capacity such as multi-states of cell, relaxing size of neighborhood with distance-decay effects, and linkage with complexity theory [10]. As the core of CA model, transition rules have also been modified and expanded to include notions such 


\section{J. Cheng and I. Masser}

as hierarchy, self-modification, probabilistic expressions, utility maximization, accessibility measures, exogenous links, inertia, and stochasticity; in fact, many-if not allurban CA bear little resemblance to the formal CA model [11]. Nevertheless, interpretation of transition rules, which is highly important for urban planners, still receives little attention in process modeling. Most studies focus on how to make complicated models.

The previous studies of urban CA models ignore the fact that urban growth is a dynamic process rather than a static pattern. Similar patterns, the final outputs of CA simulation do not indicate similar processes. Thus, the transition rules tested are not evidential to explain the complex spatial behavior. Therefore, process rather than pattern oriented simulation should be the major concern of urban growth CA modeling. This point is started to be aware in some journals [11]. In GIS field, [12] applied fuzzy spatio-temporal interpolation to simulate changes that occurred between snapshots registered in a GIS database. The main advantage of the research lies in its flexibility to create various temporal scenarios of urbanization processes and to choose the desired temporal resolution. The author also declared that the approach does not explicitly provide causal factors, thus it is not an explanatory model.

In summary, we need to take spatial and temporal process into CA modelling to achieve stronger interpretation capacities of causal factors. With this in mind, this paper is organized into four sections. Following the introduction, the next section discusses in detail a proposed methodology, which mainly comprises dynamic weighting and mathematical models of local growth. One of major features in our CA model is to utilize dynamic weighting for linking pattern and process. Sections 3 moves to the implementation of the methodology by a case study area from Wuhan City, P.R.China. Section 4 ends with some discussion and conclusions.

\section{Methodology}

As a typical self-organizing social-economic system (SOS), urban system modelling must call for an innovative bottom-up simulation approach. Complexity of urban growth comprises the multiplicity of spatial patterns and social economic processes, nonlinear interactions among numerous components and heterogeneity over a variety of spatial and temporal scales. Intuitively, the complexity of urban growth process can be transferred into spatial and temporal complexity when projected onto land system. The understanding rather than prediction of urban growth process based on SOS mechanisms is a feasible way. This understanding must be based on the integration of top-down and bottom-up approach.

As an effective bottom-up simulation tool, CA firstly offers a new thinking way for dynamic spatial modelling, and secondly provides a laboratory for testing human being's decision making. However, the complexity of urban growth determines that the classic CA must be modified in order to deal with practical issues (the details are described in [4]). In this paper, we develop a modified CA model for understanding 


\section{CA-based Temporal Process Modelling}

the spatial and temporal processes of urban growth based on dynamic weighting concept and project-based approach to be described below.

\subsection{Temporal Heterogeneity (dynamic weighting)}

[13] applied logistic regression method for modelling land development patterns in two periods (1979-1987 and 1987-1992) based on parcel data extracted from aerial photos. They found that the major determinants of land development have changed significantly, e.g. from proximity to inter-city highways to proximity to city streets. Likewise, if we shrink the long period (1979-1992) to shorter period such as 19932000 and also from the whole city to smaller part. The same principle should be working as well. As a consequence, the factors influencing local growth should be assigned with dynamic weight values.

Obviously temporal pattern from time $t_{l}$ to $t_{n}$, is influenced by highly complicated spatial and temporal processes. However, similar patterns can result from numerous different processes. As a consequence, the understanding of process is more important than that of pattern. Pattern is only a phenomena but process is the essence. The interaction between pattern and process is a non-linear iteration function like other phenomena: fractal, chaos etc. which are typically represented by non-linear iteration function (eq.1).

$$
\mathrm{X}_{\mathrm{t}+1}=\mathrm{f}\left(\mathrm{x}_{\mathrm{t}}\right)
$$

In the case of urban growth, temporal complexity might be indicated by:

- Compared with major roads, minor roads especially in new zones, which are also new development units, may have certain time delay in affecting local growth, i.e. between $T_{0}$ and $T_{n}$, not immediately from $T_{0}$;

- The spatial impacts of various factors such as road, center, rail are not simultaneous temporally in effecting local growth;

- Neighbourhood effects may suffer from temporal variation, for example, it may be stronger in $T_{0}$ than in $T_{n}$, or vice versa.

Figure 1 is only an example of temporal complexity involved in urban growth. $T_{1}, T_{2}, T_{3}$ indicate time series. The same spatial pattern results from three (in reality, more) distinguishing temporal process, which reflect the spatial and temporal interactions between road-influenced and center-based local growth. The arrows indicate the trend of temporal development, from which we can define them as three different temporal processes (convergence, sequence and divergence). The basic principle behind this phenomena is that various physical factors like road and center point take temporally varied roles in the course of local growth. In the first one, road is more important than center at time $T_{1}$, but less important at $T_{2}$. It means that local growth occurs along road first and then moves to the center. The third one takes an opposite 


\section{J. Cheng and I. Masser}

effect. If we use $D$ to denote the total amount of local growth, $D_{l}$ for the lower part along road, $D_{u}$ for the upper part along road, $D_{c}$ for the center part and $D_{t}$ for the continuous development till time $t$. Hereby, $\mathrm{D}=D_{n}=D_{l}+D_{u}+D_{c} . \mathrm{W}_{\mathrm{r}}$ and $\mathrm{W}_{\mathrm{c}}$ represent the weight value of factor ROAD and CENTER respectively. We are able to detect the following rules:

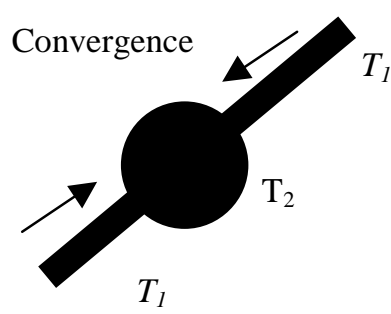

$T_{1}$

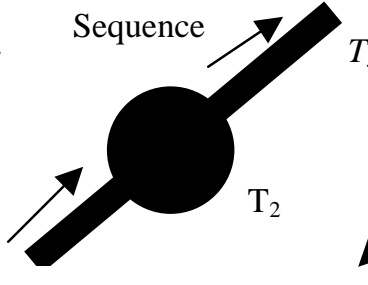

$T_{1}$

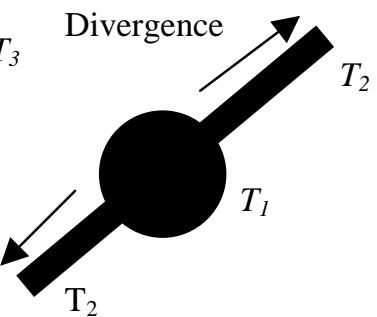

Fig.1. Temporal Heterogeneity

In Convergence: if $\mathrm{D}_{\mathrm{t}}<\mathrm{D}_{1}+\mathrm{D}_{\mathrm{u}}, \mathrm{W}_{\mathrm{r}} \rightarrow 1, \mathrm{~W}_{\mathrm{c}} \rightarrow 0$ (at $\mathrm{T}_{1}$ ); if $\mathrm{D}_{\mathrm{t}}>\mathrm{D}_{1}+\mathrm{D}_{\mathrm{u}}, \mathrm{W}_{\mathrm{r}} \rightarrow 0$, $\mathrm{W}_{\mathrm{c}} \rightarrow 1\left(\right.$ at $\left.\mathrm{T}_{2}\right)$;

In Sequence: if $\mathrm{D}_{\mathrm{t}}<\mathrm{D}_{1}, \mathrm{~W}_{\mathrm{r}} \rightarrow 1, \mathrm{~W}_{\mathrm{c}} \rightarrow 0\left(\right.$ at $\left.\mathrm{T}_{1}\right)$; if $\mathrm{D}_{\mathrm{t}}>\mathrm{D}_{1}$ and $\mathrm{D}_{\mathrm{t}}<\mathrm{D}_{1}+\mathrm{D}_{\mathrm{c}}, \mathrm{W}_{\mathrm{r}} \rightarrow 0$, $\mathrm{W}_{\mathrm{c}} \rightarrow 1\left(\right.$ at $\left.\mathrm{T}_{2}\right) ;$ if $\mathrm{D}_{\mathrm{t}}>\mathrm{D}_{1}+\mathrm{D}_{\mathrm{c}}$ and $\mathrm{D}_{\mathrm{t}}<\mathrm{D}, \mathrm{W}_{\mathrm{r}} \rightarrow 1, \mathrm{~W}_{\mathrm{c}} \rightarrow 0\left(\right.$ at $\left.\mathrm{T}_{3}\right)$;

In Divergence: if $\mathrm{D}_{\mathrm{t}}<\mathrm{D}_{\mathrm{c}}, \mathrm{W}_{\mathrm{r}} \rightarrow 0, \mathrm{~W}_{\mathrm{c}} \rightarrow 1\left(\right.$ at $\mathrm{T}_{1}$ ); if $\mathrm{D}_{\mathrm{t}}>\mathrm{D}_{\mathrm{c}}$ and $\mathrm{D}_{\mathrm{t}}<\mathrm{D}, \mathrm{W}_{\mathrm{r}} \rightarrow 1$, $\mathrm{W}_{\mathrm{c}} \rightarrow 0\left(\right.$ at $\left.\mathrm{T}_{2}\right)$.

Here, symbol " $\rightarrow$ " means "approaching to or close to". The three cases imply that the temporal complexity could be represented and understood through dynamic weighting. It means that factor weight is a function of temporal development amount, i.e.

$$
\mathrm{W}_{\mathrm{i}}(\mathrm{t})=\mathrm{f}_{\mathrm{i}}
$$

$\left(D_{t}\right)$

In principle, the function $f_{i}\left(D_{t}\right)$ should be continuous, which may be a step linear or more complicated non-linear function. In practice, the function $f_{i}\left(D_{t}\right)$ has to be simplified through discretization. It implies that the whole period needs to be divided into few cases $t_{1} \sim t_{n}$, each with varied weight values. 


\section{CA-based Temporal Process Modelling}

\subsection{Generalized mathematical models}

Urban growth process is effected by many factors, which may change their influential roles spatially and temporally. The spatial heterogeneity phenomena (heterogeneity in a spatial context means that the parameters describing the data vary from place to place) suggests that the search for general laws frequently fail in practice and it is being replaced by local area analysis like Geographically Weighted Regression (GWR) and others in the field of spatial statistics. As a consequence, a project-based local growth modelling is more reasonable for understanding of complex urban growth process. The spatial extent described below is limited to individual large-scale project.

$$
\left.\mathrm{L}(\mathrm{t})\right|_{\mathrm{t}=\mathrm{n}}=\mathrm{L}_{\mathrm{d}}(\mathrm{x}, \mathrm{y})
$$

Here, $L_{d}$ is the actual area of land development of one project $d$ in the whole period $[t=1 \sim n] . L_{d}$ in principle should result from traditional top-down socio-economic models. Here it is assumed to be a known value. $L(t)$ is the simulated area of land development of same $d$ till time $t$. $L(t)$ will be calculated from the later section. As an example, we only refer to project $d$; the others follow the same procedures.

$$
\mathrm{C}_{\mathrm{i}}(\mathrm{t})=\mathrm{f}_{\mathrm{i}}(\mathrm{L}(\mathrm{t}))
$$

Here, $C_{i}(t)$ is the temporal contribution of factor $i$ to $L(t)$ at time $t$, which is a function rather than a subjective parameter. We strongly argue that $C_{i}$ should vary with $L(t) . W_{i}(t)$ in eq. 5 is a relative weight of factor $i\left(0\left[W_{i}(t)[1)\right.\right.$.

$$
W_{i}(t)=\frac{C_{i}(t)}{\sum_{i} C_{i}(t)}
$$

To some extent, eq. 4 and 5 indicate a dynamic feedback between $w_{i}(t)$ and $L(t)$, which can be utilized to represent the complex interactions between pattern and process. Actually, $L(t)$ is to quantify temporal pattern, and the process can be described by multiple $w_{i}(t)$. The non-linear iteration function $f(L(t))$ ( together with $L(t)=g\left(W_{i}(t)\right.$ ) exhibit the complexity property of the interactions between pattern and process like 'chicken and egg'. When $f_{i}(L(t))$ is constant, $C_{i}$ is becoming universe temporally, which is treated in most CA applications, however, it can not model the temporal process. The design of function $f_{i}(L(t))$ is a critical point. It needs numerous experimental tests, which is based on the theoretical understanding of the interaction "chicken and egg". However, model from the experimental tests with higher temporal resolution are able to theoretically abstract the hidden temporal rules. The development potential of each cell $j$ at time $t$ is defined as: 


$$
P_{j}(t)=\left(1+\ln (\xi)^{\alpha}\right)\left(\sum_{i=1}^{k} W_{i}(t)^{*} V_{i j}(t)\right) \prod_{i=k+1}^{m} \omega_{i}
$$

Assuming that totally $m$ constraints $(1 \leq i \leq m)$ are considered, when $k+1 \leq i \leq m, \omega_{1}$ (binary variable: 0 or 1) are restrictive constraints such as water body, slope etc, which may include local, regional and global levels with equal weight.

When $1 \leq i \leq k$, they are non-restrictive constraints. $W_{i}(t)$ is the weight value of constraint $i$ computed from eq.5. For proximity variables like the distance to major road, here a negative exponential function is employed to calculate $V_{i j}(t)$. Urban models based on economic theory [14], and discrete choice theory [15] had made widespread uses of the negative exponential function.

$$
\mathrm{V}_{\mathrm{ij}}(\mathrm{t})=\mathrm{e}^{-\phi \mathrm{dij}} \quad 0<\mathrm{V}_{\mathrm{ij}}(\mathrm{t})<1
$$

$d_{i j}$ is the value of proximity variable $i$ at cell $j . \phi$ is the density gradient for quantifying its spatial influence. Usually, $0<\phi<1$, and $\phi$ varies with factor $i$. Eq. 7 is actually to standardise non-restrictive variables.

In order to generate the patterns that are closer to reality, a stochastic disturbance is introduced as $\left(1+\ln (\xi)^{\alpha}\right)[16]$. $\xi$ is a random variable within [0 1]. $\alpha$ is a parameter controlling the size or strength of the stochastic perturbation. $P(t)$ represents the potential or probability of development of cell $j$ at time $t$, which is the major driving force of local growth.

In our model, neighbourhood size is not universal globally and is locally parameterised, which varies with different projects. Neighbourhood effect i.e. 'action-atdistance' is also represented as one or two non-restrictive variables in eq.6, which indicate the spatial influences of developed cell (including both the new and the old) on its surrounding sites.

$$
\begin{gathered}
\mathrm{P}_{\mathrm{j}}(\mathrm{t}) \rightarrow \mathrm{P}_{\mathrm{j}^{\prime}}(\mathrm{t}) \\
\Delta \mathrm{L}(\mathrm{t})=\mathrm{L}(\mathrm{t})-\mathrm{L}(\mathrm{t}-1), \quad \mathrm{L}(0)=0
\end{gathered}
$$

Eq.8 builds a transition from $P_{j}(t)$ to $P_{j^{\prime}}(t)$, which is to reorder from maximum to minimum. $\Delta L(t)$ in eq. 9 is the land development demand at the phase from $t-1$ to $t$ as $L(t)$ is the accumulative amount of land development till time $t$. 


\section{CA-based Temporal Process Modelling}

Based on equation 8 and 9 , the state of cell $j$ at time $t+1$ can be determined as follows:

$$
S_{j}(t+1)= \begin{cases}1 & \text { if } P_{j}(t)=P_{j^{\prime}}(t) \text { and } j^{\prime} \in[1 \sim \Delta L(t)] \\ 0 & \text { if not }\end{cases}
$$

Simply, totally $\Delta L(t)$ cells will be selected at time $t$ for the transition from developable land (0) to urban (1) according to their development potential values $P_{j^{\prime}}(t) . L(t)$ is to be determined as below.

Another advantage of project-based CA modelling is able to control the temporal development pattern of each project. Previous studies suggest that urban development process $(L(t)$ in eq.4) follows a logistic curve over time [17]. The logistic curve is illustrated as eq. 11 .

$$
L(t)=1 /\left(a+b * e^{\left(-c^{*} t\right)}\right)
$$

Assuming that $L(0)=L_{0}=1 /(a+b)=1, L(n)=L_{n}=1 /\left(a+b e^{(-c n)}\right)=L_{d}$, the parameters $a$ and $b$ can be calculated as the functions of parameter $c$ (eq.12):

$$
a=1-b \quad b=\frac{1-\ln }{\ln \left(e^{-c n}-1\right)}
$$

The shape of logistic curve usually represents the speed of urban development over time, which is controlled by the parameter $c$ and $n$. Here, in simplicity, temporal control is classified as three types: slow growth, normal or basic growth and quick growth, which indicates three distinguishing scenarios (eq.13). Of course, you can define more classes or even use fuzzy logic.

Quick growth: $c *_{n}>25$

Basic growth: $c^{*} n<25$ and $>15$

Slow growth: $c * \mathrm{n}<15$

The selection of temporal control pattern is a top-down process of decision-making as shown in equation 14. Where $y$ denotes the real time-year $(1 \sim m)$ such as 1993 $(y=0)$ and $2000(y=7)$, which is different from iteration number $t(1 \sim n)$ in simulation.

$$
G(y)=\Sigma L_{d}(y) \quad y \leq m
$$




\section{J. Cheng and I. Masser}

$G(y)$ denotes the total growth of the whole study area till year $y, L_{d}(y)$ represents the total growth of only project $d$ till year $y$. The assignment of $L_{d}(y)$ should be determined from a top-down social-economic model. Eq.14 also offers a link between local growth and global development. It is a feedback between top-down and bottom-up decision-making.

$$
L_{i}(y)=h\left(L_{i}(t)\right) \quad y=1,2, \ldots m ; \quad t=1,2, \ldots, n ; n>m
$$

Eq.15 establishes a transition from $L_{i}(t)$ to $L_{i}(y)$. In the previous researches of CA application, a linear function is applied, i.e. $t=\Delta^{*} y$. Here $\Delta$ is assumed to be a constant, which means equal growth rate. In reality, function $h$ could be a non-linear function of iteration number $t$, which can be tested experimentally through visual exploration.

\section{Implementation}

\subsection{Case study}

Wuhan is the largest mega city in central China. In1999, it had around 4 million nonagricultural population, 4 times more than that of 1949. During the last 5 decades, Wuhan underwent rapid urban growth from 3000 ha of built-up area in 1949 to 3,0151 ha in 2000. As a result, Wuhan is a fresh and typical case for understanding the dynamic process of Chinese cities.

With the assistance of topographic maps of 1993 and SPOT Pan/Xs images of 2000, we found that land cover change in the period 1993-2000 was dominated by strong spatial agglomeration of a few large-scale projects, which take over $60 \%$ of total change. As a consequence, the understanding of local growth process of each project is highly crucial to that of whole study area. Here, Zukou car manufacturing center, the largest project, is taken as a case study for testing the methodology proposed. The influential factors include major roads, minor roads, master planning, physical constraints from water body. The cell size in this research is $100 \times 100 \mathrm{~m}^{2}$.

\subsection{CA Simulation}

The validation of parameters has been proven difficult for urban CA modelling $[6,16]$ in particular when factors and parameters considered are voluminous. Here, we think that manual test is much quicker and also more interpretable, which is based on the modeler's reasonable understanding of urban growth process and visual exploration of model outputs. The impact of each factor or parameter is assessed by changing its value and holding the others constant. In this case study, the major parameters include 


\section{CA-based Temporal Process Modelling}

"distance to minor road" (OR), "distance to major road"(MR), "distance to center"(CN), "density of neighbouring new development" (DN), " OR density gradient", "MR density gradient", "CN density gradient", and "Master planning". So their relative importance (weight values) could be assigned quantitatively by manual test, further improvement can be done by limited number of automatic search like 1000 iterations.

Model accuracy depends on measure approach to comparing simulated and actual patterns. [6] chose four ways to statistically test the degree of historical fit (three rsquared fits and one modified Lee-Sallee shape index). The last one is a measurement of spatial fit between the simulated and the actual growth. Supposed that the actual is denoted by set $\mathrm{A}$, the simulated $\mathrm{B}$; the index is equal to $(\mathrm{A} \cap \mathrm{B}) /(\mathrm{A} \cup \mathrm{B})$ mathematically. This simple measure of shape was computed through counting the union and the intersection of their total areas on a pixel x pixel basis, and then dividing the intersection by the union. For a perfect match, the Lee Sallee measure gives a value of 1.0, and for all others ranging from 0 to 1 . Clark reported the practical accuracy of his model is only 0.3 [6]. Other measures like fractal and Moran I index are also frequently used for global pattern comparison e.g.[18]. In this paper, we use consistency co-efficient $(C C)$ (spatial match between the simulated and the actual) and Lee-Sallee index $(L I)$ for goodness of fit evaluation. Mathematically, $\mathrm{CC}$ is equal to $(\mathrm{A} \cap \mathrm{B}) / \mathrm{A}$. As the total number of pixels is set the same for the simulated as the actual, apparently here $L I=C C /(2-C C)$. Following this formula, the Lee-Sallee index of Zuankou is computed and listed in table 1 . The model accuracy is $55 \%$ in $C C$ and $39 \%$ in $L I$, which is greater than Clark's [6].

Assisted with SPOT images of 1995, 2000 and IRS images of 1997, we are able to judge the temporal development pattern of Zuankou, compared with other parts of Wuhan city. In 1993, Zuankou was still completely rural and nearly half constructed in 1995. There was not much change from 1997 and 2000. So its temporal growth pattern is defined as "Quick". The number of iteration is defined as $50(n=50)$ as principally the greater the number is, the finer discriminative capacity the model has, which results in higher accuracy. Therefore, when $\mathrm{c}=0.5, \mathrm{c} * \mathrm{n}=25$. As described in equation 15 , the result of simulation is $L_{i}(t)$, which is different from yearly actual amount $L_{i}(y)$. We need a transition from $L_{i}(t)$ to $L_{i}(y)$. In simplicity, we just use equal time interval, i.e. a linear function: $y=t / 7$. As $t$ ranges from 1 to 50 and $y$ is from 1 to $7, L_{i}(y)=\Sigma L_{i}(t)(t$ from $7 *(y-1)+1$ to $7 * y)$. A new layer with 7-year urban growth (from 1993 to 2000) is input into animation software for dynamic exploration. This animation is helpful for comparing the distinguishing temporal development processes of various projects. 


\section{J. Cheng and I. Masser}

Table 1. Test of temporal heterogeneity (Zuankou)

\begin{tabular}{lllll}
\hline Results & Model 1 & Model 2 & & \\
\hline Total cells & 1390 & 1390 & & \\
Accuracy (CC) & $55 \%$ & $55 \%$ & & \\
Lee-Sallee Index & $38 \%$ & $38 \%$ & & \\
Stochastic & $1 \%$ & $1 \%$ & & \\
( $\alpha=10 \%)$ & & & & \\
Iteration number & 50 & 50 & & 0.05 \\
Neighb. size & 6 & 6 & & 0.15 \\
c & 0.5 & 0.5 & & 0.5 \\
Temporal division & $100 \%$ & $<15 \%$, & $15 \%-50 \%$, & 0.15 \\
Major road (MR) & 0.2 & - & 0.5 & 0.15 \\
Minor road (OR) & 0.3 & - & 0.1 & $100 \%$ \\
Centers (CE) & - & 0.7 & - & \\
Neighb. (new) & 0.3 & 0.3 & 0.1 & \\
Master Planning & 0.2 & - & 0.3 & \\
Total & $100 \%$ & $100 \%$ & $100 \%$ & \\
Gradient for MR & 0.0005 & 0.0005 & & \\
Gradient for OR & 0.002 & 0.002 & & \\
Gradient for CE & - & 0.0004 & & \\
\hline
\end{tabular}

Note( "-" :0)

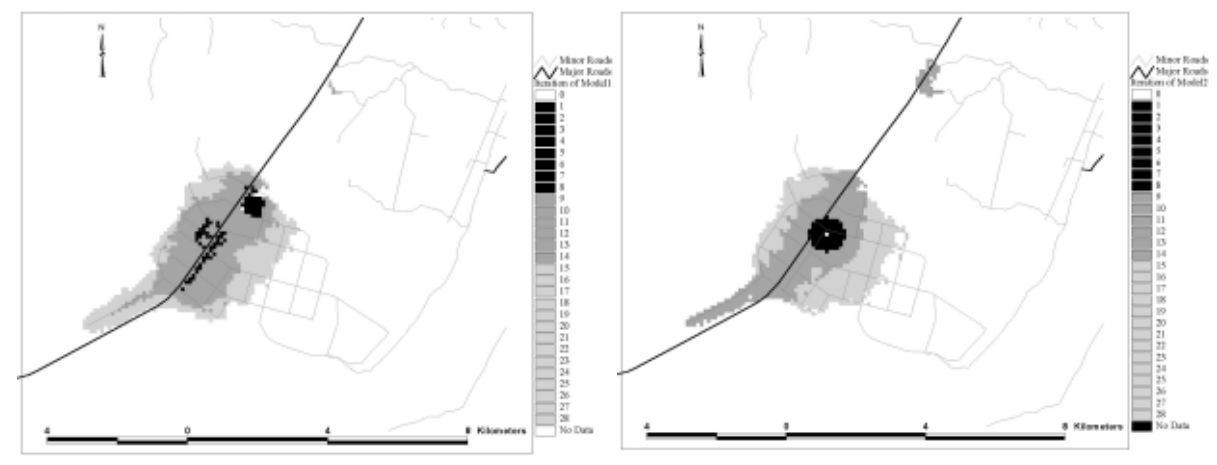

Fig.2. Test of temporal heterogeneity (Model 1 \& Model 2)

Two models of Zuankou in Table 1 have similar model accuracy and also similar pattern (the CA model is over till the $28^{\text {th }}$ step). However, their temporal processes shown in Figure 2 are quite different. The mode of temporal control is set the same $(\mathrm{c}=0.5)$. Model 1 exhibits a more random process. Model 2 shows a more organized process. Model 2 is based on the assumption that new development in Zuankou first occurred in the center, then along the major road and finally spread from the center. The assumption corresponds to a temporal process that is spatially controlled to by three sets of weight values (Table 1). In other words, the temporal process can enable us better understand the organized local growth. If we explore the changes of weight values, it can be found that the major changes are indicated in major road and center. 


\section{CA-based Temporal Process Modelling}

As explained in section 2 (eq. 3 and 4), weight values should be the functions of temporal development demand. Table 1 also shows the functions are highly complicated in reality. A universal or standard function is not available. Rather it should be simplified and based on local knowledge. Model 2 actually is based on the interviews with local planners.

\section{Discussion and Conclusions}

Although the accuracy of two CA models is only $55 \%$, simulation model accuracy, to some extent, depends on the complexity and stochasticity of real city and also the availability of more detailed information. From the previous part of this research, the accuracy of global pattern model that is based on logistic regression analysis with 10 explanatory variables is only around $70 \%$. If more detailed data like control plan scheme is available, more rigorous model calibration will become possible. From the angle of spatial modelling, as criticized by other researchers, CA is not an appropriate tool on micro scale, we need to integrate agent-based techniques e.g.[19]. Models of complex systems with geographic properties, such as city and ecology systems, usually involve spatial and temporal processes, which are difficult to embed within proprietary GIS. Most CA software available such as AUGH, DUEM either lack GIS functions or do not fit specific complex city. A loose coupling strategy is still preferred, which is also adopted in this research.

We can not ignore the fact that any advanced modelling techniques including CA must be based on the proper understanding and abstract of the system studied. The more proper, the more accurate it is. The ability of science to understand the real world is to a large extent dependent on knowledge constrained by the limits of our understanding of complexity.

CA is only a simulation tool for testing user's understanding. Limited by existing GIS theory and technique, the identification of spatial and temporal heterogeneity can not be completed without the assistance of local knowledge as rich historical data layers do not guarantee the improvement of model calibration. It implies that local knowledge is an important ancillary data sources for CA modelling. During modeling, temporal control, dynamic weighting, and manual test need more local knowledge. For the division of temporal process, due to limited temporal resolution, local knowledge is a key source of qualitative information.

The major purpose of CA simulation is to generate alternative scenarios for decision support in a smart growth management. Apparently, the methodology developed here can be extended in this direction. As it is based on the soft systems thinking, which stresses the role of users' subjectivity; Local planners' intention can be transformed into spatially and temporally explicit weight values and certain parameters. 


\section{References}

1. Xie, Y. and Batty, M., Automata-based exploration of emergent urban form. Geographical System, (1997) 83-102.

2. Allen, P.M., Cities and regions as evolutionary, complex systems. Geographical systems, (1997) 103-130.

3. Batty, M. and Torrens, P.M., Modeling complexity: the limits to prediction. CyberGeo, (2001) .

4. Couclelis, H., From cellular automata to urban models: new principles for model development and implementation. Environment and Planning B, (1997) 165-174.

5. Batty, M., Xie, Y., and Sun, Z., Modelling urban dynamics through GIS-based cellular automata. Computers, Environment and Urban Systems, (1999) 205-233.

6. Clarke, K.C. and Gaydos, L.J., Louse-coupling a CA model and GIS: long-term urban growth prediction for San Franciso and Wanshington/Baltimore. Interlational Journal of Geographical Information Science, (1998) 699-714.

7. White, R. and Engelen, G., High resolution integrated modelling of the spatial dynamics of urban and regional systems. Computers, Environment and Urban systems, (2000) 383-440.

8. Yeh, A.G. and Xia, L., A constrained CA model for the simulation and planning of sustainable urban forms by using GIS. Environment and Planning B, (2001) 733 753.

9. Ward, D.P., Murray, A.T., and Phinn, S.R., A Stochastically constrained cellular model of urban growth. Computers, Environment and Urban Systems, (2000) 539558.

10. O'Sullivan, D., Graph-cellular automata: a generalised discrete urban and regional model. Environment and Planning B, (2001) 687-705.

11. Torrens, P.M. and O'Sullivan, D., Editorial: Cellular automata and urban simulation: where do we go from here? Environment and Planning B, (2001) 163-168.

12. Dragicevic, S., Marceau, D.J., and Marois, C., Space, time, and dynamics modeling in historical GIS databases: a fuzzy logic approach. Environment and Planning B, (2001) 545- 562.

13. Wu, F. and Yeh, A.G.-O., Changing spatial distribution and determinants of land development in Chinese cities in the transition from a centrally planned economy to a socialist market economy: a case study of Guangzhou. Urban Studies, (1997) 18511879.

14. Muth, R., Cities and Housing: The Spatial Pattern of Urban Residential Land Use, Chicago University Press, Chicago, IL. (1969)

15. Anas, A., Residential Location Markets and Urban Transportation: Economic Theory, Econometrics and Policy Analysis with Discrete Choice Models, Academic Press, New York (1982)

16. Li, X. and Yeh, A.G.-O., Calibration of cellular automata by using neural networks for the simulation of complex urban systems. Environment and Planning A, (2001) 1445-1462.

17. Herbert, D.T. and Thomas, C.J., Cities in Space: City as place, 3nd edn, David Fulton Publishers, London (1997)

18. $\mathrm{Wu}, \mathrm{F}$., An experiment on the generic polycentricity of urban growth in a cellular automata city. Environment and Planning B, (1998) 731-752.

19. Bernard, R.N., An application of agent-based modelling to planning policy: the case of rent control, in Department of urban planning and policy development, Rutgers University, (1999) 